\title{
Patient Perceptions and Social Impact. Preliminary Results of the Bristol MRC Study
}

\author{
K. J. LOWE, D. A. GREGORY, R. I. JEFFERY, D. L. EASTY \\ Bristol
}

\begin{abstract}
Summary
One hundred and nine inpatients were compared with 84 day cases by means of specially designed questionnaires presented at set times by staff other than the operating surgeon. The aim was to highlight patient attitudes, expectations and satisfaction with a standard method of endocapsular cataract extraction and posterior chamber lens implant under local anaesthetic as either a day case (DC) or an inpatient (IP). The results showed a high patient acceptance of whichever method of management was chosen. Both groups appeared satisfied with their treatment and the final result. The cost of DC and IP treatment was assessed.
\end{abstract}

Cataract extraction with posterior chamber lens implant is regarded as an effective treatment with a satisfactory outcome. However clinical impressions of patient satisfaction and actual patient satisfaction may differ as shown for unilateral cataract extraction with spectacle correction. ${ }^{1}$

Few attempts to assess patient satisfaction with cataract extraction have been documented in the UK and these have not involved lens implants. ${ }^{2,3}$ Reports from the USA on patient satisfaction with DC cataract surgery refer to small numbers of patients who were specially selected. They are significantly different in both age and social background from the cataract population of the UK. ${ }^{4}$

We set out to compare DC and IP management for cataract surgery by measuring our patients' opinions of their treatment and the final result. This approach enabled us to undertake a useful comparison between the two groups. It also assessed IP management in a way which to date has rarely been attempted.

\section{Materials and Methods}

The first 109 in patients and 84 day cases are presented. All patients were recruited from the Bristol Eye Hospital waiting list, based on the inclusion and exclusion criteria listed (Tables I, II).

Patients were invited by letter to attend a preoperative assessment clinic. This outlined the clinical trial and stated that allocation to each treatment group would be random. Patients who attended the clinic and agreed to be included in the trial were then told which group they were in. History and examination including biometry were undertaken in the assessment clinic.

Patients were prepared for surgery within sedation. Posterior peribulbar anaesthesia was used for all cases. A standardised endocapsular cataract extraction with posterior chamber lens implant was performed by the same surgeon. Stay in hospital was the only variable between the DC and IP groups.

DCs were admitted at 12 noon and discharged around $5.30 \mathrm{pm}$. They were visited at

From: University Department of Ophthalmology, Bristol Eye Hospital.

Correspondence to: Mr. K. J. Lowe, University Department of Ophthalmology, Bristol Eye Hospital, Lower Maudlin Street, Bristol BS1 2LX. 
Table I. Inclusion criteria for recruitment clinic

Inclusion criteria

On one of 6 consultant waiting lists

Aged 55 or over

Living within an 8 mile radius of hospital

Informed consent

Table II. Exclusion criteria for recruitment clinic

Exclusion criteria

Listed for GA

Listed for procedure other than extraction with lens implant

Listed for consultant only

Previous intraocular procedure on same eye

home on the first two post-operative mornings by the ophthalmic nurse. A portable slit lamp and Tono-pen 2 (strain gauge tonometer) were used during the examination. The IPs were admitted the day before surgery and discharged on the second morning after their operation. Both groups attended clinic at 2, 8 and 16 weeks after operation.

We designed a series of questionnaires to assess patient attitudes towards, expectations of and satisfaction with cataract surgery. These were carefully worded to avoid bias. They provided a method of assessing the patients views and our success in patient education.

Questionnaires were presented preoperatively by a social worker, two days postoperatively by the ophthalmic nurse (DC only) and about 16 weeks postoperatively by a research assistant. The results were analysed

Table III. Response of patients to recruitment letter

\section{Recruitment clinic attendance}

\begin{tabular}{lr}
\hline Attended clinic & $379(75 \%)$ \\
DNA & $51(10 \%)$ \\
Refused trial & $42(8 \%)$ \\
Postponed & $25(5 \%)$ \\
Deceased & $5(1 \%)$ \\
Wrong address & $4(1 \%)$ \\
\hline
\end{tabular}

Table IV. Ability to put in own drops

\begin{tabular}{lccc}
\hline Put in own drops post-op & Yes & Difficult & No \\
\hline DC & $25 \%$ & $54 \%$ & $21 \%$ \\
IP & $36 \%$ & $42 \%$ & $22 \%$ \\
\hline
\end{tabular}

using Chi-squared or Fisher's exact probability.

\section{Results}

Of the 590 patients selected as suitable for inclusion from the waiting lists, 84 were excluded when their hospital records were checked by the surgeon leaving 506 (84\%). The major reasons for exclusion were GA was required, there had been previous intraocular surgery on the same eye, there was coexistent eye pathology or there was a significant medical or psychiatric history. Of the 506 patients sent for $51(10 \%)$ ignored the request to attend the clinic, a further $76(15 \%)$ did not attend for the reasons outlined (Table III). Of the 379 who attended 374 were suitable for inclusion in the trial. The rejections were due to: one macular hole; one disciform macular degeneration; one retinal detachment and two insignificant lens opacities.

So far 109 IPs and 84 DCs have completed their treatment and follow up. The results are presented as percentages.

\section{Composition}

Females outnumbered males by almost $2: 1$ in each group. The mean age of DCs was 73.7 years, range 55-99 years. The mean age of IPs was 74.6 years, range $55-92$ years.

\section{Understanding and expectations}

Preoperatively $87 \%$ of DCs felt that a cataract extraction with lens implant was a minor to intermediate procedure compared to $82 \%$ of IPs. Only $13 \%$ of DCs and $18 \%$ of IPs felt it was a major procedure. Visual expectations were the same for DCs and IPs, $99 \%$ expected an improvement, $1 \%$ expected no change, no one expected a deterioration.

\section{Support}

In the DC group $19 \%$ of males and $55 \%$ of females lived alone. In the IP group $22 \%$ of males and $56 \%$ of females lived alone. Eightyseven per cent of DCs and $92 \%$ of IPs felt they would be able to look after their eye after the operation. They were less confident about drop instillation (Table IV). Eighty-five per cent of DCs and $83 \%$ of IPs expected help to be available from friends or relatives after operation. 


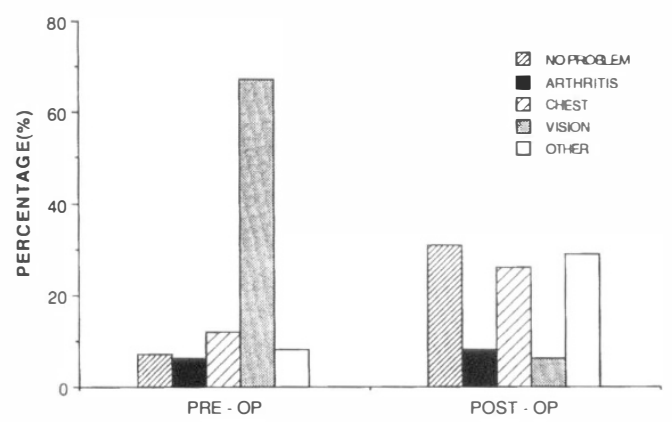

Fig. 1. Aspect of health which affected patients most, before and after operation. Day cases.

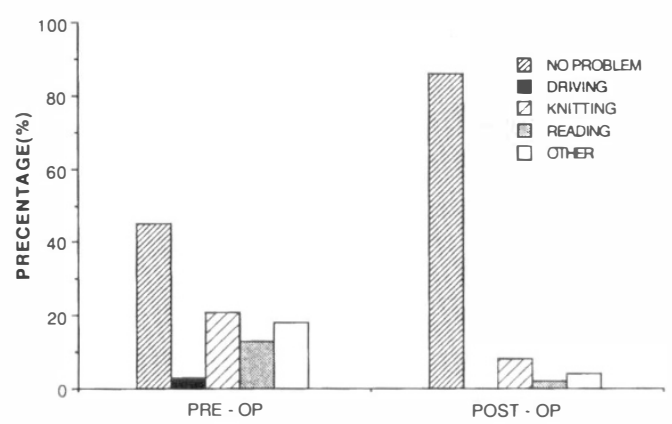

Fig. 3. Hobbies patients found difficult due to their eyesight, before and after operation. Day cases.

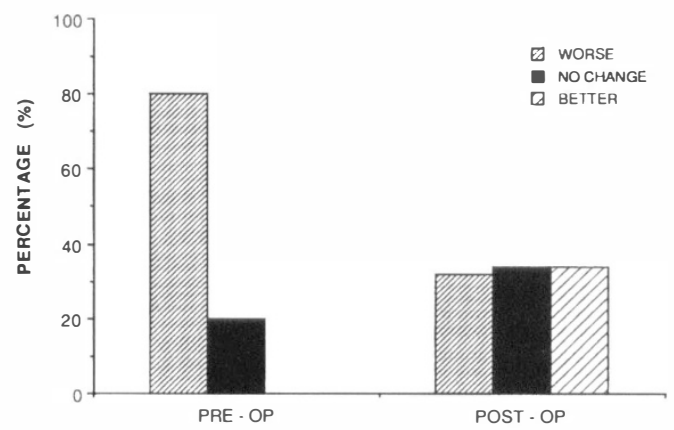

Fig. 5. Affect of bright sunlight on vision, before and after operation. Day cases.

Patients were asked the following questions before and 16 weeks after operation.

(1) Which aspect of their health affected them the most (Fig. 1,2).

(2) To list hobbies they found difficult because of their eyesight (Fig. 3, 4).

(3)* Whether bright sunlight affected their vision (Fig. 5, 6).

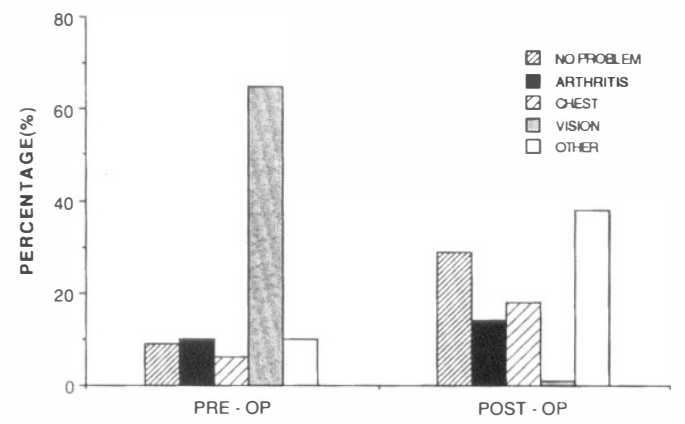

Fig. 2. Aspect of health which affected patients most, before and after operation. Inpatients.

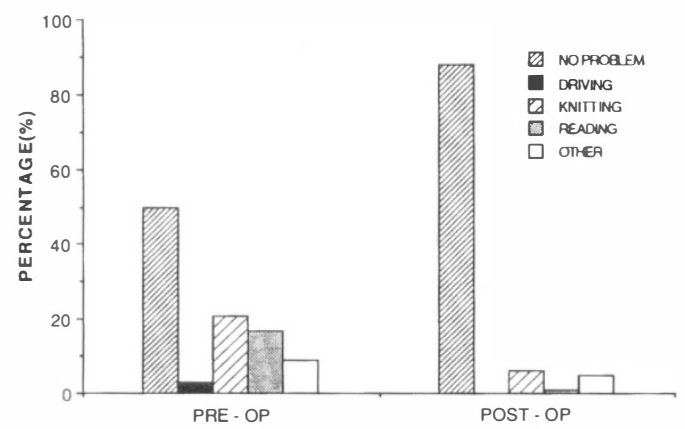

Fig. 4. Hobbies patients found difficult due to their eyesight, before and after operation. Inpatients.

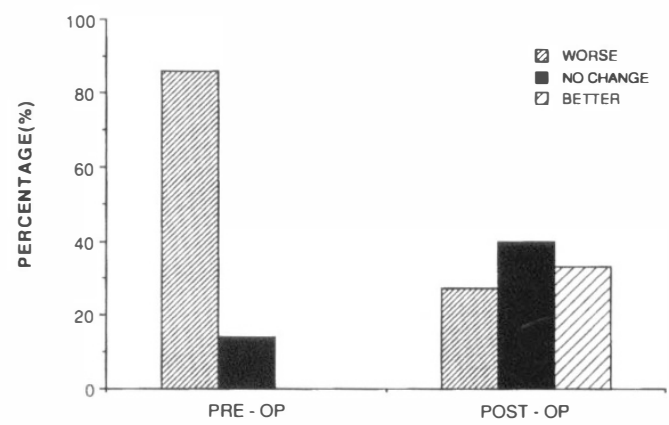

Fig. 6. Affect of bright sunlight on vision, before and after operation. Inpatients.

(4)* Whether they felt in danger because of their eyesight (Fig. 7, 8).

(5)* Given a choice would they have chosen GA, LA or did they have no preference (Fig. 9, 10).

(6)* Given a choice would they have chosen to be a DC, IP or did they have no preference (Fig. 11, 12). 


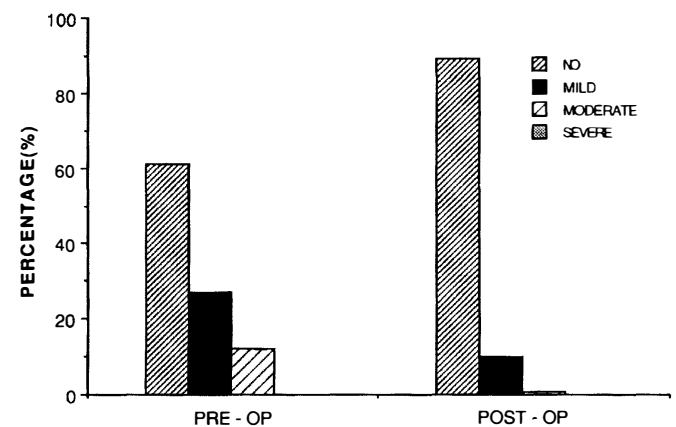

Fig. 7. Felt in danger because of eyesight, before and after operation. Day cases.

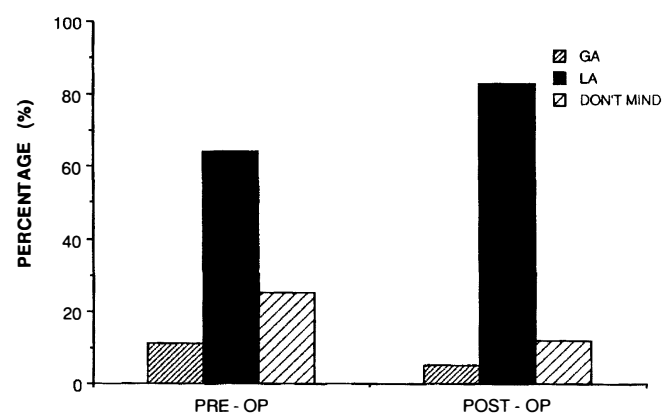

Fig. 9. Patient preference for type of anaesthesia, before and after operation. Day cases.

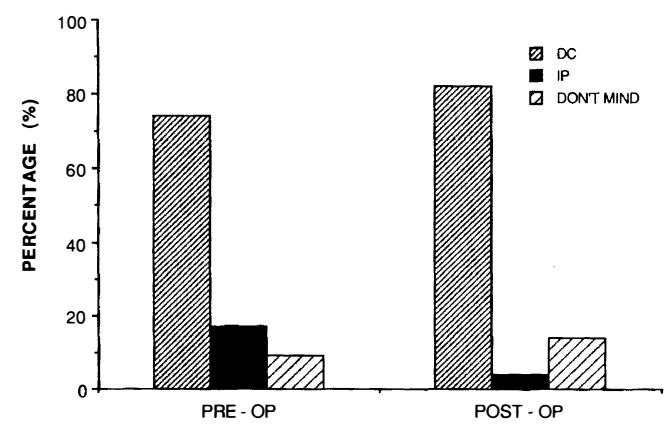

Fig. 11. Patient preference for DC or IP treatment, before and after operation. Day cases.

*(Figures show overall groups, $\mathrm{p}$ value calculations based on choice of individual patients).

\section{Visual changes}

Vision in bright sunlight improved after operation $(\mathrm{p}<0.001)$ for DC and IP groups. Comparing DC with IP before and after operation showed no significant difference.

The number of patients who felt in danger

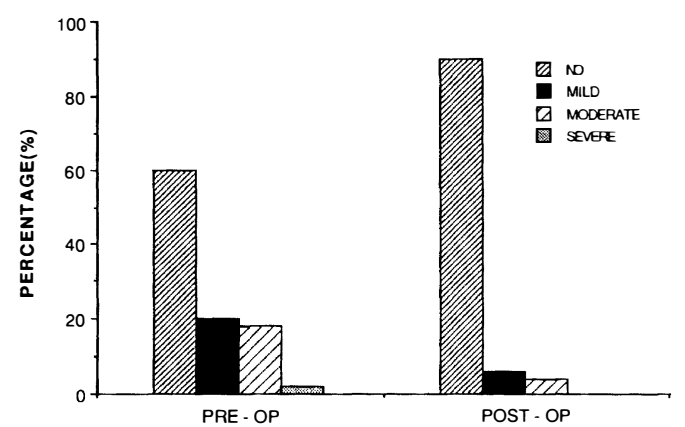

Fig. 8. Felt in danger because of eyesight, before and after operation. Inpatients.

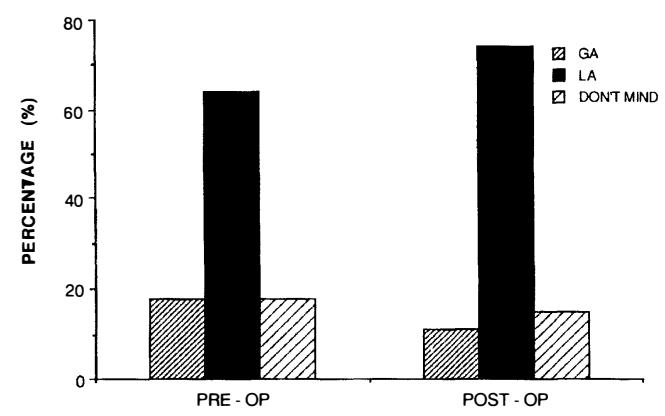

Fig. 10. Patient preference for type of anaesthesia, before and after operation. Inpatients.

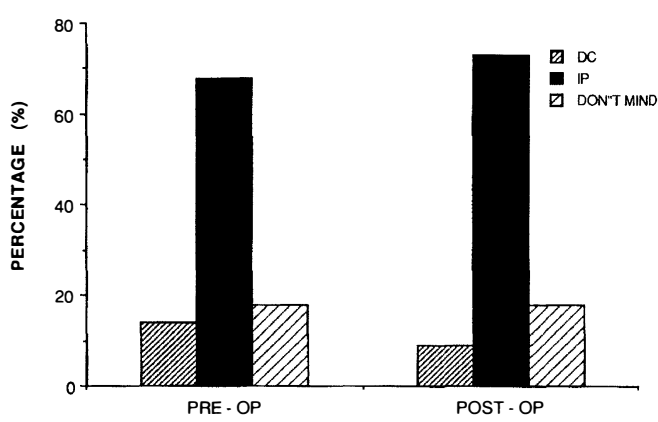

Fig. 12. Patient preference for DC or IP treatment, before and after operation. Inpatients.

due to their eyesight was reduced $(p<0.001)$ comparing before and after operation for DC and IP groups. Comparing DC with IP groups showed no significant difference before or after operation.

\section{Preference for anaesthetic}

In the DC group before operation $90 \%$ were content to have LA and $10 \%$ preferred a GA. Afterwards $2 \%$ still preferred a GA and $2 \%$ 
Table V. Who provided help on the first two postoperative days. Day cases only

Source of help for DC days 1 and 2 Post-op

\section{Spouse}

Son/Daughter

Brother/Sister

Other

No help

$36 \%$

$16 \%$

$5 \%$

$23 \%$

$20 \%$

Table VI. Ability to attend hospital on the first postoperative day. Day cases only

\begin{tabular}{lccc}
\hline Question & Yes & No & With help \\
\hline $\begin{array}{l}\text { Well enough to attend } \\
\text { hospital first day }\end{array}$ & $55 \%$ & $4 \%$ & $41 \%$ \\
Arrange own transport & $57 \%$ & $2 \%$ & $41 \%$ \\
\hline
\end{tabular}

Table VII. Cost of DC and IP surgery

Revenue assessment of DC and IP cataract surgery

\begin{tabular}{lcc}
\hline & DC $(£)$ & IP (£) \\
Assessment clinic & 34 & - \\
Admission & 14 & 34 \\
Operation & 325 & 325 \\
Post-op recovery & 90 & - \\
Ward care (3 days) & - & 300 \\
Home visit (2) & 40 & - \\
Outpatient attendance & 102 & 102 \\
\hline Total & 605 & 761 \\
\hline
\end{tabular}

*(Cost of post-op recovery is included as part of ward care for inpatients)

changed their preference to GA, leaving $96 \%$ satisfied with LA. In the IP group before operation $81 \%$ were content to have LA and $19 \%$ preferred a GA. Afterwards $8 \%$ still preferred a GA and $3 \%$ changed their preference to GA, leaving $89 \%$ satisfied with LA. This was not significant comparing preference for LA before and after operation, nor was there a significant difference between DC and IP groups before or after operation.

\section{Preference for DC or IP treatment}

In the DC group before operation $87 \%$ were content to be DCs and $13 \%$ preferred to be IPs. Afterwards $2 \%$ still preferred to be an IP and $1 \%$ changed preference to IPs, leaving $97 \%$ satisfied as DCs. In the IP group before operation $86 \%$ were content to be IPs and $14 \%$ preferred to be DCs. Afterwards 5\% still preferred to be a DC and 5\% changed preference to DC, leaving $90 \%$ satisfied as IPs. The preference for type of treatment is significant $(p<0.001)$ comparing DCs with IPs before and after operation. Preference within each group does not change significantly comparing before and after operation.

\section{Satisfaction with treatment}

In the DC group $100 \%$ were satisfied with the explanation of their operation, and the care before and after surgery. In the IP group 99\% were satisfied with the explanation of their operation and $100 \%$ with the care before and after surgery.

\section{Satisfaction with visual result}

In the DC group 97\% were pleased with their vision, $1 \%$ had no view and $2 \%$ were disappointed. In the IP group $96 \%$ were pleased with their vision, $2 \%$ had no view and $2 \%$ were disappointed. When asked if they would still have had their operation 16 weeks ago knowing the result $99 \%$ of DCs and $97 \%$ of IPs said yes.

Day cases

In the first two days after the operation $32 \%$ put in their eye drops. Eighty per cent had some kind of help (Table V), 52\% felt they needed help. Nearly everyone felt well enough to attend hospital on the first morning after operation, however only half could be sure of transport (Table VI). None of our DCs preferred to come to hospital for their first dressing, $89 \%$ preferred a home visit and $11 \%$ had no preference.

The running costs of DC or IP surgery based on the method outlined are assessed (Table VII).

\section{Discussion}

The majority of adult patients within the urban area around Bristol listed for cataract surgery seem suitable for either type of management. The response to the trial was generally favourable and would be higher as part of a normal service. Some patients were not happy to be involved in a trial.

With the exception of preference for DC or IP surgery attitudes expectations and satisfaction were not significantly different between the groups by any of the parameters we measured. This was true before and after surgery. 
The outside environment, particularly traffic was the main situation when patients felt in danger due to their vision. Expectations of visual improvement was almost universal. After operation eyesight was rarely the main problem, ability to pursue hobbies improved and bright sunlight was less troublesome. This suggests most patients were achieving 'useful' changes in vision.

It is interesting that although vision in bright light was improved overall, about one third of patients still had some difficulty. This did not correlate with opacities in the other eye. We do not have any data on the prevalence of this problem in aged matched normals.

Patients had been told that all operations would be performed under local anaesthetic. Both DC and IP groups showed a preference for local anaesthesia. The DC patients showed a preference for day case surgery, but the IP showed a preference for inpatient surgery. This difference between DCs and IPs was significant before and after operation. Whether these results are due to a belief that the doctor will recommend the most appropriate treatment or due to a willingness to accept any available treatment is uncertain. As a group the elderly adapted well to either treatment. The fact that most would opt for the same type of treatment again suggests that it is both effective and satisfactory.

We deliberately kept our exclusion criteria to a minimum to avoid treating a highly selected group. Some of our DC patients lived in nursing homes or sheltered accommodation, which meant they had good support. These patients would have been excluded had we been selecting for active self supporting people. Most women and some men lived alone, nevertheless they managed. Most people felt they could cope though many were unsure about putting in drops. Drop instillation is a potential problem in DCs and IPs. In practice it proved less of a problem than expected largely due to support from relatives friends and neighbours. Another problem which affected both groups was transport.

Patient education was very important. The days when more than a week was spent in bed after cataract extraction and restrictions were imposed on activity for a long period after are still remembered. We were able to allay many of our patient's fears about surgery and convalescence. With instruction and encouragement from our nurse most who needed to instil their own drops managed. On one occasion the help of a district nurse was required twice a day.

The DC group seemed happy to be treated at home rather than in hospital. The Home visits proved popular and patients readily accepted examination and instruction from the nurse. The majority felt they could have attended hospital for the first examination if necessary. This would be important if a day case service was planned on an outpatient basis.

There was a high level of satisfaction with the care given at all stages and with the outcome of surgery. The continuity of care was probably a significant factor in patient satisfaction. Patients repeatedly stated that it was reassuring to see the same faces, especially on the day of surgery.

The cost of DC surgery was $20 \%$ less than a three day stay in hospital. However as IP stay is reduced the difference may ultimately be small.

We are grateful to the finance department of the Bristol and Weston Health Authority for the costings of cataract surgery.

This work was funded by the Medical Research Council, grant No. G8621834. We would also like to acknowledge the Bristol Eye Hospital League of Friends and the NERC (National Eye Research Council) for their support.

Key words: Cataract extraction, Patient attitudes, Expectation, Satisfaction.

\section{References}

${ }^{1}$ Hilbourne JFH: Social and other aspects of adjustment to single eye cataract extraction in elderly patients. Trans Ophthalmol Soc UK 1975, 95: 254-9.

${ }^{2}$ Ingram RM, Banerjee D, Traynar MJ, Thompson RK: Day-case cataract surgery. Trans Ophthalmol Soc UK 1980, 100: 205-9.

${ }^{3}$ Ingram RM, Banerjee D, Traynar MJ, Thompson RK: Day-case cataract surgery. Br J Ophthalmol 1983, 67: 278-81.

${ }^{4}$ Smith S: Day case cataract surgery. The patients perspective. J Ophthalmic Nurs Technol 1987, 2: 50-6. 